\title{
A Study of Self-regulated Learning in an Online Gaming Environment for Engineering Education
}

\author{
Gayatri Mehta, Brett Kellerstedt, Jeanne Tunks, Kiran Sanagapaty, Alok Pal, and Rani Deepika \\ Balavendran Joseph
}

\begin{abstract}
In this paper, we study the self-regulated learning aspect in an interactive open-ended game-like design environment. We used the interactive game UNTANGLED to conduct this study. This paper is focused on studying the effect of time breaks on the performance of game players and the quality of solutions players generate. Our results suggest that i) games should require a certain level of metacognition, so that players are expected to self-evaluate, self-direct, and self-select choices that lead to feasible solutions; ii) game should include motivational tools that aid in the movement through the material available in the game that leads to meaningful learning; and iii) games should enhance behaviors that lead to various strategies players can select from for decision making.
\end{abstract}

Index Terms-Engineering education, games with a purpose, mapping, placement, self-regulated learning, STEM games.

\section{INTRODUCTION}

Single player electronic game platforms, particularly those with educational purposes, by design require self-regulated learning (SRL). Players set goals, adjust strategies based on feedback, employ motivating factors associated with the game, to achieve the successful completion of the game. These aspects of game play align with the study of self-regulated learning posted by Zimmerman [1], [2]. He found that self-regulated learners are metacognitively, motivationally, and behaviorally active participants in their learning. Metacognitively SLRs set goals, organize, self-monitor, and self-evaluate. Motivationally SRLs use feedback to adjust goals and monitor progress toward accomplishing goals. Behaviorally SRLs actively seek strategies that lead to goal attainment. Assessment of the SRL was done in the form of self-reported answers on questionnaires regarding three aspects: motivation, metacognition, and behavior toward task completion.

Reference [3] expanded the theoretical position of SRL and introduced the notion of self-efficacy as an additional component of SRL. He noted that self-efficacious learners, in addition to self-regulation, entered the learning environment

Manuscript received March 23, 2016; revised May 18, 2016. This work has been supported by the National Science Foundation under grants CCF-1117800 and CCF-1218656.

Gayatri Mehta is with Electrical Engineering, University of North Texas, Texas, USA (e-mail: gayatri.mehta@unt.edu).

Brett Kellerstedt and Jeanne Tunks are with College of Education, University of North Texas, Texas, USA (e-mail: Brett.Kellerstedt@unt.edu, jeanne.tunks@unt.edu).

Kiran Sanagapaty, Alok Pal, and Rani Deepika Balavendran Joseph are with College of Engineering, University of North Texas, USA (e-mail: KiranSanagpaty@my.unt.edu,

RaniDeepikaBalavendranJoseph@my.unt.edu). as self-observers, self-judgers, and self-reactors. As self-observers, learners observe their practice and adjust according to the progress made toward goals. As self-judgers learners constantly compare their current performance against self-selected goals. Finally, self-reactors enhance self-efficacy when they believe that if progress is acceptable, satisfaction toward goal accomplishment motivates progress. Schunk contends that even negative feedback does not deter self-reactors if they believe they are capable of reaching their goal.

These two early authors set the stage for other studies that tested the theoretical position [4]-[9]. Winne and Perry introduced the concept of Trace. The development and subsequent study of gStudy, a computer based learning environment, designed to teach a myriad of concepts, includes in the structure, a method for Tracing learners' motivation, metacognition, and behavior as they progressed through learning in gStudy. This real-time, as opposed to post-hoc approach to ascertaining the aspects of SRLs is purported to more accurately represent the aspects of SRL. Sabourin et al. studied learners as they progressed through an electronic learning tool Crystal Island, using social media checkpoints throughout the SRL decision-making eighth grade students were making regarding a medical emergency on the island. Their findings showed that SRLs were more likely to find a so lution by using the motivational, metacognitive and behavioral tools available in the game. Lower SRLs overlooked the tools and strategies available and did not reach conclusions equal to the Higher SRLs who did employ the tools. These findings suggest that theory of SRL is maintained, but that new technologies and gaming platforms offer a new perspective on how the characteristics of SRLs are determined in learning environments.

\section{A. Phenomena}

The current paper makes use of the UNTANGLED game environment designed by Mehta and colleagues [10] to conduct the study. Twenty-one game puzzles, ranging from easy to hard, across three types of games, were created to ascertain how well independent SRLs would resolve the game. The games are related to electrical engineering concepts such as exploring low power architectures for portable electronics. Each game was designed to provide electrical engineering researchers with human-engineered solutions to the puzzles presented in the games. All games had a Trace aspect to them. Each player's self-selection of type, frequency, and timing or play was traced and saved in the database. Although the original purpose of this interactive gaming environment was to gather and analyze players' mapping solutions to discover 
novel and efficient mapping algorithms for low power portable electronics, a phenomenon of self-regulated learning emerged.

Trace data suggested that the use of time gaps, stops in the game play, showed that some players stopped play at times, suggesting a metacognitive consideration before play continued. Analysis of trace data suggested distinctions among players who self-monitored and adjusted play toward goal attainment. Although these data did not collect data in the form of social media status reporting, member checks for metacognition, or Likert scales regarding self-regulation, the data show patterns of success among self-regulators who persisted, used motivation, and achieved success in the form of puzzle solutions.

\section{B. Purpose}

The purpose of this study was to answer the following research questions:

- Does the number of breaks players take during game play predict total score?

- Does the average time per break during game play predict the total score?

- Do break takers get higher scores than those that do not take breaks?

\section{Hypothesis}

We hypothesize that the players who take frequent time breaks perform better, reach good solutions, and achieve higher scores as compared to those who do not take time breaks.

\section{BACKGROUND}

UNTANGLED received the "People's Choice Award" in the Games \& Apps category of the 2012 International Science and Engineering Visualization Challenge conducted by the National Science Foundation and Science. It is designed to be accessible to broad audience and players do not require any kind of engineering background to play. The game has in-depth tutorials that provide good hands-on experience to the players. The game puzzles are arranged according to the level of difficulty and are categorized as easy, medium, and hard levels of difficulty. The game also has a leaderboard where players can keep track of their scores and rankings in comparison to other players worldwide. Players also get incentives such as badges and medals during the game play. The game has been continuously online since May 2012 and can be played from the website https://untangled.unt.edu/. The details of the game can be found in [10] and more information about how crowds solve problems can be found in the following books [11]-[13].

\section{EXPERIMENTAL SET-UP}

In this section, we describe our experimental set-up and methods used to conduct the study presented in this manuscript. The experimental protocol for all studies was determined to qualify for an exemption from the Institutional Review Board of the University of North Texas. IRB protocols were followed in all cases.

\section{A. Games Considered in This Study}

In this paper, we present results for three games: 8Way, 4Way1Hop, and 4Way2Hops shown in Fig. 1. 8Way is a game where blocks can connect to any of their 8 neighbors. 4Way1Hop is a game allowing connectivity to direct horizontal and vertical neighbors, as well as horizontal and vertical connections that skip one node. $4 \mathrm{Way} 2 \mathrm{Hop}$ is a game that allows horizontal and vertical connections that skip two nodes.

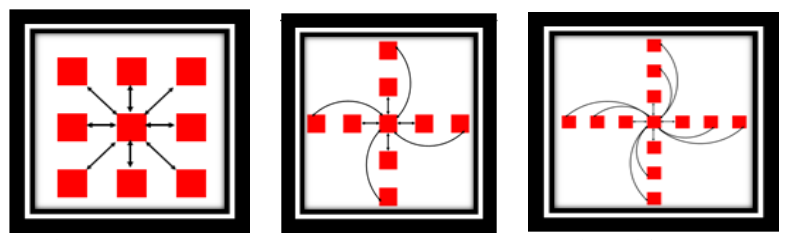

Fig. 1. 8Way, 4Way1Hop, and 4Way2Hops mesh architectures.

\section{B. Benchmarks}

The seven benchmarks considered in this study are taken from signal and image processing domain. These are arranged according to the level of difficult and categorized as easy, medium and hard puzzles. These include E1, E2, E3, M1, M2, $\mathrm{H} 1$ and $\mathrm{H} 2$. Basic statistics are shown in Table I.

TABLE I: BASIC INFORMATION RELATED TO THE BENCHMARKS

\begin{tabular}{llllllll} 
Benchmarks & E1 & E2 & E3 & M1 & M2 & H1 & H2 \\
\hline $\begin{array}{l}\text { Number of } \\
\text { Blocks }\end{array}$ & 24 & 29 & 29 & 29 & 36 & 52 & 61 \\
$\begin{array}{l}\text { Number of } \\
\text { Connections }\end{array}$ & 29 & 29 & 34 & 36 & 53 & 63 & 72 \\
\hline
\end{tabular}

\section{Obtaining Participation}

UNTANGLED has been online since May 2012. A worldwide online game competition was conducted in 2012 , which took place for a period of ten days from August 10 to August 20. In order to get good participation from players, a variety of resources were used for advertising such as online press releases and university-sanctioned posts on social networking websites. Current players were also encouraged to participate in the competition. After the competition, the game has remained online and continued to attract new and returning players. To date, overall more than 800 players have played several games provided on UNTANGLED website, who have completed more than 11800 mapping solutions. Players used random user names or self-selected login names to play the games. Information about the participants' such as their background, demographics, age, etc. was not saved in the database. The only information we consider is how players' play and solve the game puzzles. As we had mentioned earlier, our study was determined to qualify for an exemption from the Institutional Review Board of our university. For the competition, gift card incentives were provided to winners of each architecture and overall competition. Rankings were visible on the UNTANGLED leaderboard throughout the competition and can still be viewed on the game website https://untangled.unt.edu/competition/home.php. In the results reported here, we use all played games, including those contributed after the end of the competition. In total, there were 1748 solutions for the 21 game puzzles considered in this study. 
TABLE II: QUESTION ONE REGRESSION COEFFICIENTS

\begin{tabular}{|c|c|c|c|c|c|c|c|}
\hline & $\begin{array}{c}\text { Unstandardized } \\
\text { Coefficients }\end{array}$ & & $\underline{\text { Standardized Coefficients }}$ & & & $95.0 \%$ Confi & nce Interval for \\
\hline & B & Std. Error & B & $\mathrm{T}$ & $P$ & $\begin{array}{l}\text { Lower } \\
\text { Bound }\end{array}$ & Upper Bound \\
\hline Intercept & 146619.317 & 3162.431 & & 46.363 & .000 & 140414.360 & 152824.273 \\
\hline Number of Breaks & 4087.714 & 383.466 & .304 & 10.660 & .000 & 3335.321 & 4840.106 \\
\hline
\end{tabular}

TABLE III: QUESTION TwO REGRESSION COEFFICIENTS

\begin{tabular}{|c|c|c|c|c|c|c|c|}
\hline & $\begin{array}{l}\text { Unstandardized } \\
\text { Coefficients }\end{array}$ & & $\underline{\text { Standardized Coefficients }}$ & & & \multicolumn{2}{|c|}{$\begin{array}{l}95.0 \% \text { Confidence Interval for } \\
\text { B }\end{array}$} \\
\hline & B & Std. Error & B & $\mathrm{t}$ & $p$ & $\begin{array}{l}\text { Lower } \\
\text { Bound }\end{array}$ & Upper Bound \\
\hline Intercept & 181677.989 & 4001.670 & & 45.401 & .000 & 173821.852 & 189534.125 \\
\hline Average Break Time & -8.052 & 11.721 & -.025 & -.687 & .492 & -31.062 & 14.958 \\
\hline
\end{tabular}

TABLE IV: DESCRIPTIVE STATISTICS BY GROUP

\begin{tabular}{|l|l|l|l|l|l|l|}
\hline & & & & & \multicolumn{2}{c|}{$95 \%$ Confidence Interval for Mean } \\
\hline & $\mathrm{N}$ & \multicolumn{1}{|c|}{ Mean } & SD & Std. Error & Lower Bound & Upper Bound \\
\hline No Breaks & 88 & 140065.91 & 64229.387 & 6846.876 & 126457.00 & 153674.82 \\
\hline Break Takers & 259 & 216017.80 & 84722.949 & 5264.428 & 205651.08 & 226384.52 \\
\hline Total Sample & 347 & 196756.22 & 86514.628 & 4644.349 & 187621.52 & 205890.93 \\
\hline
\end{tabular}

\section{Method}

Trace data from all trials of 21 UNTANGLED game puzzles were configured for analysis using multiple statistical procedures. Results from the analyses were used to examine the aspects of self-regulated learning theory that applied to the findings.

\section{E. Data Analysis}

In order to address all three research questions, separate regression and ANOVA analysis were employed after aggregating and recoding data. All coding and analysis was performed in SPSS.

First, time between intervals was calculated by subtracting time stamps for each individual response, creating a latency score for reach response. Here we coded the existence of a break before that particular response. If the latency was greater than 30 seconds and less than an hour then a break was marked for that response. Also, if a break was marked, the latency was also recorded into another variable as a measure of break time before that response.

A new data set was created by aggregating individual response data into cases by an individual player, on an individual date. These recorded the date, user, and game type. They also calculated maximum score in session, sum of violations, average latency and total latency. Then number of breaks and average break time were also aggregated by session.

Finally, in order to address research question three, a third dataset was created with each user as a case. This dataset carried over the user id of each user and calculated maximum score, total violations, average latency between responses, total latency between responses, total number of breaks and average break amount. Then they were coded as takers if they took any breaks, and non-takers if they were not.

To answer research question one, "does the number of breaks predict total score," a bi-variate regression was performed. Number of breaks by session was regressed onto the maximum score by session. To answer research question two, "does the average time per break predict the total score," another bi-variate regression was performed. Average break time for sessions that took breaks was regressed onto the maximum score by session. To answer research question three, "do break takers get higher scores than those that do not take breaks" a one way ANOVA was performed comparing the Non break taking group to the break taking group in terms of top total score.

\section{RESULTS}

The number of breaks taken in a session statistically significantly predicted maximum score within session $(t=10.66, p>.001)$. In this model, number of breaks shared $9 \%$ of the variance in maximum score within session $\left(R^{2}=0.092\right)$. Table II shows standardized and unstandardized coefficients along with confidence intervals for unstandardized coefficients. Fig. 2 shows a scatter plot of our top scores by the number of breaks. The average time per break within session did not statistically significantly predict maximum score within session $(t=-0.687, p=0.492)$. Average time shared less than $0.1 \%$ of variance with maximum score within session. Table III shows standardized and unstandardized coefficients along with confidence intervals for unstandardized coefficients.

Break takers had a much higher maximum score then non test takers $(d=0.946)$. This difference was statistically significant $(F=59.128, p>0.001)$ but since the groups were 
not homogenous in variance (Levine's Statistic $=6.629$, $p=0.01$ ) then Welch's statistic was used to determine statistical significance (Welch's $F=77.335, p>0.001$ ). There was no difference, what so ever between a Welch's ANOVA and Brown-Forsyth ANOVA (Brown Forsyth $F=77.335$, $p>0.001)$. Table IV shows descriptive statistics by group, including confidence intervals and Fig. 3 shows a simple whisker plot showing plausible population mean values by group.

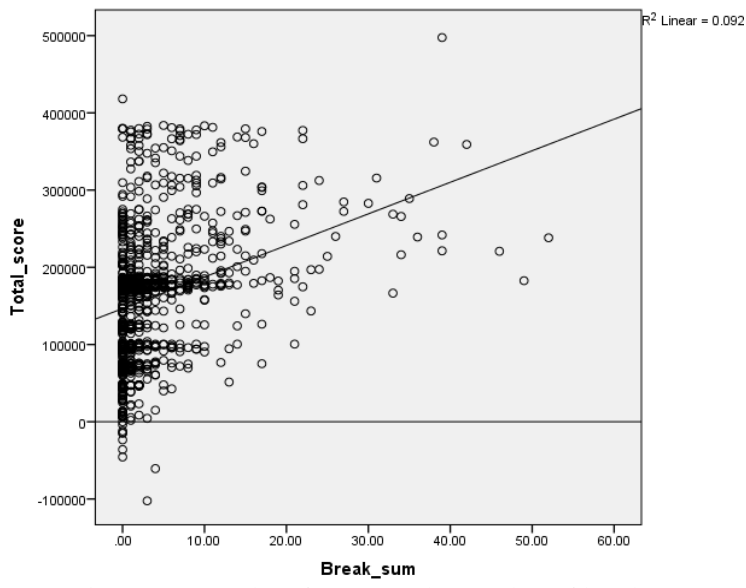

Fig. 2. Scatter plot of top score by number of breaks.

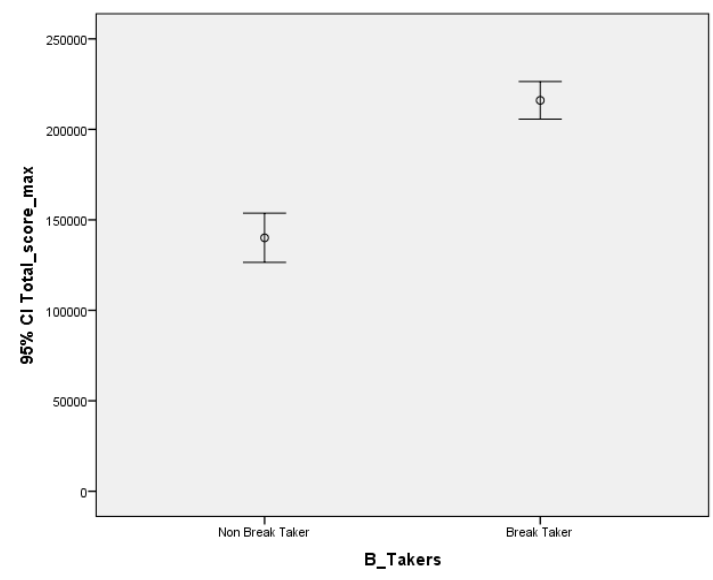

Fig. 3. Means by group with standard errors.

\section{DISCUSSION}

The study of time gap analysis, using trace data available to game designers, supported the theoretical position that high-level self-regulated learners use strategies, motivation, and adjustments to achieve goals. The distinction between players of Untangled who reached a solution and those who did not are observed in the findings from the study of time gap. Players who used time as a strategy to contemplate, reconsider, and adjust play decisions resulted in significantly higher scores and reached solutions more frequently than players who did not use time as a strategy to make adjustments The findings suggest that metacognition was an important part of the success of the higher performing, self-regulated learners.

According to Zimmerman [1], metacognition requires goal setting, organization, self-monitoring, self-evaluation, self-selection, structure and creating environments that optimize learning. By taking time away from the games, successful solutions were evident among high-performing players. The findings from this study support the importance of the metacognitive decision to use the strategy of time away from the game in achievement. The distinction between non-achievers of solutions and achievers is shown in the choices made by players who elected to take time, contemplate, and adjust. These findings support the metacognitive aspect of self-regulated learning as found in trace data collected during game play.

Although motivation was not examined specifically in this study, all players were provided with two motivational tools: points and violations. As play persisted after breaks, scores increased, hence violations decreased. A zero sum violation was an indicator of correct solution to the puzzle. Players who took breaks used the two motivational tools either before or after breaks to change play sequences that resulted in higher scores and subsequent success in finding a solution. In contrast, those who did not take breaks or used fewer breaks, did not benefit from the motivational tools of points and violations, as observed in the lower points and higher violations of this group.

The behavior of taking a break supports the third aspect of self-regulated learners. Reference [1] points out that actions and processes directed at acquisition of learning, in the case of this study, game solution, "involve agency, purpose, and instrumentality; distinguished by awareness of strategies and use of these". The use of time gaps was purposeful, as noted by the use of time relief by highly successful players who reached a solution to the games. The strategy served as a meaningful, valuable tool employed by self-regulated learners who intentionally chose to stop, think, and proceed. The lower performing SRLs did not apply the behavior of using the strategy of time gap, therefore were not as successful.

\section{IMPLICATIONS FOR EDUCATION AND CONCLUSION}

Studies of SRL in education point out that support for self-regulated learning, either electronically or from teachers, is paramount to success [8], [9], [14], [15]. In the learning game environments designed by Winne, Parry and Sabourin, SRLs report their status as they work through the learning/gaming environment. Tsai points out that the combination of SRL and problem based learning, when monitored by instructors resulted in higher levels of learning. These initial studies suggest that SRL can be enhanced in gaming/learning environments, particularly when trace data are collected.

These laudable findings lead to implications for both electronic platforms for learning as well as learning taught in face-to-face classrooms. Game designers are encouraged to design games that relate to the aspects of self-regulated learning defined by Zimmerman in [1]. First, games should require a certain level of metacognition, so that players are expected to self-evaluate, self-direct, and self-select choices that lead to feasible solutions. Second, game should include motivational tools that aid in the movement through the material available in the game that leads to meaningful learning. Finally, games should enhance behaviors that lead to various strategies players can select from for decision making. 
Throughout all game play, trace data that shows these three aspects of SRL in a meaningful way.

Teachers who are using games in classrooms to enhance learning should be circumspect about which games encourage self-regulated learning. It would be important for teachers to understand the three aspects of self-regulated learning and know when they are studying a game to bring into the classroom, and whether these games support or deny the aspects. Since there is currently no such evaluative tool for teachers to use when selecting a game platform, it would be important for teachers to study the work of SRL researchers and use the tenets of their findings for guidance in selecting educational electronic gaming platforms.

In education, every learner is a self-regulated learner, of some degree. However, the tenets of the theory of self-regulated learning posited by Zimmerman and colleagues serves as a clear mandate to examine the self-regulation of learning to determine if metacognition, motivation, and behaviors that emulate growth and development are present, and at what level. These tenets, coupled with self-efficacy, the belief that you can learn, when employed in educational settings, can change a quiet, self-regulated compliant setting of learning into a vibrant, exuberant, engaged setting when the aspects of self-regulated learning are monitored and supported.

\section{ACKNOWLEDGEMENTS}

We would like to thank the National Science Foundation for supporting this work with grant NSF CCF-1117800 and NSF CCF-1218656. We would like to acknowledge Ashwin Tonge and Xiaozhong Luo for their contributions to this work We would like to thank all the UNTANGLED players.

\section{REFERENCES}

[1] B. J. Zimmerman, "Self-regulated learning and academic achievement: An overview," Educational Psychologist, vol. 25, no. 1, 1990, pp. 3-17.

[2] B. J. Zimmerman, "Investigating self-regulation and motivation: Historical background, methodological developments, and future prospects," American Educational Research Journal, vol. 45, no. 1, pp 166-183, March 2008.

[3] D. H. Schunk, "Goal setting and self-efficacy during self-regulated learning," Educational Psychologist, vol. 25, no. 1, 1990, pp. 71-86.

[4] P. Nenniger, "Commentary on self-regulation in the classroom: A perspective on assessment and intervention," Applied Psychology, vol. 54, no. 2, pp. 239-244, April 2005.

[5] N. E. Perry, L. Phillips, and L. Hutchinson, "Mentoring student teachers to support self regulated learning," The Elementary School Journal, vol. 106, no. 3, pp. 237-254, 2006.

[6] P. H. Winne, "Improving measurement of self-regulated learning," Educational Psychologist, vol. 45, no. 4, pp. 267-276, 2010.

[7] N. E. Perry and P. H. Winne, "Learning from learning kits: gStudy traces of students' self-regulated engagements with computerized content," Educational Psychology Review, vol. 18, no. 3, pp. 211-228, Sep. 2006.

[8] C. W. Tsai, "An effective online teaching method: The combination of collaborative learning with initiation and self-regulation learning with feedback," Behaviour \& Information Technology, vol. 32, no. 7, pp. 712-723, July 1, 2013,

[9] J. L. Sabourin, L. R. Shores, B. W. Mott, and J. C. Lester, "Understanding and predicting student self-regulated learning strategies in game-based learning environments," International Journal of Artificial Intelligence in Education, vol. 23, no. 1-4, pp. 94-114, Nov. 2013.

[10] G. Mehta et al., "Untangled: A game environment for discovery of creative mapping strategies," ACM Transactions on Reconfigurable Technology and Systems (TRETS), vol. 6, no. 3 p.13, 2013.

[11] C. R. Sunstein, Infotopia: How Many Minds Produce Knowledge, Oxford University Press, 2006.

[12] J. Surowiecki, The Wisdom of Crowds, Anchor, 2005.

[13] P. Michelucci, Handbook of Human Computation, Springer, 2013.

[14] P. H. Winne, "How software technologies can improve research on learning and bolster school reform," Educational Psychologist, vol. 41, no.1, pp. 5-17, 2006.

[15] N. E. Perry, C. Thauberger, and L. Hutchinson. "gStudy traces of children's self-regulated learning in the lifecycles learning kit," Psychological Test and Assessment Modeling, vol. 52, no. 4, pp. 432-453, 2010.

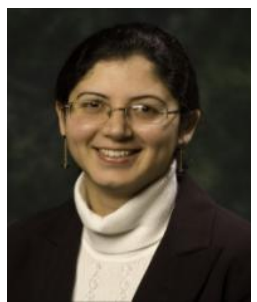

computing.
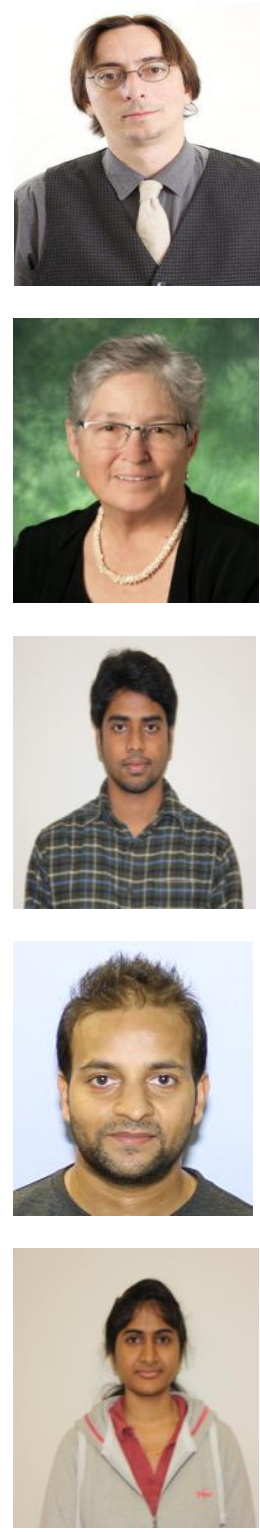

Gayatri Mehta Gayatri Mehta is an associate professor in the Department of Electrical Engineering at the University of North Texas. She received her $\mathrm{Ph} . \mathrm{D}$ in electrical and computer engineering from the University of Pittsburgh in 2009. Her research interests are broadly in the areas of reconfigurable computing, low-power VLSI design, system-on-a chip design, electronic design automation, embedded systems, portable/wearable

Brett Kellerstedt is a 3rd year PhD student in the Educational Psychology Department with a concentration in research measurement and statistics. His areas of interest are research methodology and experimental design, especially single subject research design, but he also has interests in statistical methods and measurement.

Jeanne Tunks is an associate professor in the College of Education, with specialization in curriculum and instruction and educational research. She currently serves as director of the core curriculum for the University of North Texas.

Kiran Sanagapaty did his master's in computer Science and engineering from the University of North Texas. He worked in the reconfigurable computing lab at UNT under the mentorship of Dr. Gayatri Mehta.

Alok Pal is working towards his master's degree in computer science and engineering at the University of North Texas. He works in the reconfigurable computing lab at UNT under the mentorship of Dr. Gayatri Mehta.

Rani Deepika Balavendran Joseph is currently working towards her $\mathrm{Ph} . \mathrm{D}$ in electrical engineering at the University of North Texas. She is working in the reconfigurable computing lab at UNT under the mentorship of Dr. Gayatri Mehta. 